\title{
ON DESCARTES-HARRIOT'S RULE ${ }^{1}$
}

\author{
PAUL TURÁN
}

The classical rule of Descartes-Harriot asserts that if

$$
f(x)=a_{0}+a_{1} x+\cdots+a_{n} x^{n}
$$

is a polynomial with real coefficients, then the number $P$ of its positive zeros cannot exceed the number $V_{0}$ of the sign changes of the sequence

$$
a_{0}, a_{1}, \cdots, a_{n}
$$

(if some of the coefficients vanish they can be omitted). This rule has been extended and refined in various ways. $V_{0}$ yields obviously an upper bound for the number $P^{*}$ of zeros lying in $0<x<1$; then Laguerre ${ }^{2}$ observed the $P^{*}$ is majorised also by the number $V_{1}$ of sign changes of the sequence

$$
a_{0},\left(a_{0}+a_{1}\right), \cdots,\left(a_{0}+a_{1}+\cdots+a_{n}\right),\left(a_{0}+\cdots+a_{n}\right), \cdots
$$

which is not greater than $V_{0}$. More generally $P^{*}$ is majorised by $V_{k}$, where $V_{k}$ denotes the number of sign changes of that sequence which arises from (2), completing it with zeros to an infinite sequence and forming the sequence of $k$ times iterated partial sums; these $V_{k}$ 's form a nonincreasing sequence. One trend of the investigations is the study of the $V_{k}$ for large $k$; this was done mainly by M. Fekete and G. Pólya. ${ }^{3}$ Another trend is to obtain similar rules representing $f(x)$ in various other forms (Runge, ${ }^{4}$ Sylvester, ${ }^{5}$ Obreschkoff, ${ }^{6}$ I. J. Schoenberg $^{7}$ ) or even generally for the linear combinations

$$
h(x)=a_{0} \phi_{0}(x)+\cdots+a_{n} \phi_{n}(x)
$$

Received by the editors June 22, 1948.

1 To the memory of my late friend, Ervin Feldheim.

2 E. Laguerre, Mémoire sur la théorie des équations numériques, J. Math. Pures Appl. (3) vol. 9 (1883) pp. 99-146.

${ }^{3}$ See in particular their joint paper in Rend. Circ. Mat. Palermo vol. 34 (1912) pp. 89-120 entitled Über ein Problem von Laguerre.

4 See the paper of G. Polya, Über einige Verallgemeinerungen der Descartesschen Zeichenregel, Archiv der Mathematik und Physik vol. 23 (1915) pp. 22-32.

5 J. J. Sylvester, Mathematical papers, vol. 2, pp. 360 and 401.

${ }^{6} \mathrm{~N}$. Obreschkoff, Über die Wurzeln algebraischer Gleichungen, J. Deutschen Math. Verein vol. 33 (1924) pp. 52-64.

${ }^{7}$ I. J. Schoenberg, Zur Abzählung der reellen Wurzeln algebraischen Gleichungen, Math. Zeit. vol. 38 (1934) pp. 546-564. 
of a prescribed system $\phi_{0}(x), \phi_{1}(x), \cdots\left(\right.$ Pólya-Szegö ${ }^{8}{ }^{8}$ S. Bernstein $\left.{ }^{9}\right)$. A further trend is the comparison of the different rules (F. Klein, ${ }^{10}$ I. J. Schoenberg ${ }^{7}$ ), extensions permitting complex coefficients in (1) and substituting the segments of the positive axis by more general domains (Obreschkoff, ${ }^{11}$ I. J. Schoenberg ${ }^{12}$ ), and finally the improving the rule by suitable Tschirnhausen-transformation (S. Lipka ${ }^{13}$ ). But as far as we know no similar rule has been given to a lower estimation of the number $P$ of the positive roots. The aim of this note is to give such a simple rule. This is based on the Laguerre-polynomialrepresentation of $f(x)$ :

$$
f(x)=\sum_{\nu=0}^{n} b_{\nu} L_{\nu}(x)
$$

$b_{\nu}$ real,

where the Laguerre-polynomials $L_{\nu}(x)$ are defined as is well known by

$$
e^{-x} L_{\nu}(x)=\frac{1}{\nu !} \frac{d^{\nu}}{d x^{\nu}}\left(e^{-x} x^{\nu}\right)
$$

The rule we are going to prove will assert that the number $P$ of positive roots of $f(x)$ is not less than the number of sign changes in the sequence of successive differences of the coefficients $b_{\nu}$, that is, in the sequence

$$
\begin{gathered}
b_{0},\left[b_{0}-b_{1}\right],\left[b_{0}-2 b_{1}+b_{2}\right], \cdots, \\
{\left[b_{0}-C_{n, 1} b_{1}+C_{n, 2} b_{2}-\cdots+(-1)^{n} C_{n, n} b_{n}\right] .}
\end{gathered}
$$

It is perhaps not uninteresting to notice that if $\eta_{n}$ denotes the greatest positive root of $L_{n}(x)$, then ${ }^{6}$ the number of sign changes of the coefficient-sequence

$$
b_{0}, b_{1}, \cdots, b_{n}
$$

itself furnishes an upper bound for the number of the roots of $f(x)$ in

${ }^{8}$ G. Pólya and G. Szegö, Aufgaben und Lehrsätze aus der Analysis, vol. 2, chap. 5, probleme 87-90.

${ }^{9} \mathrm{~S}$. Bernstein, Leçons sur les propriêtés extremales et la meilleure approximation des fonctions analytiques d' une variable reelle, Paris, 1926.

${ }^{10} \mathrm{~F}$. Klein, Geometrisches zur Abzählung der reellen Wurzeln algebraischer Gleichungen, Gesammelte Mathematische Abhandlungen, pp. 198-208.

${ }^{11}$ N. Obreschkoff, Sur un problème de Laguerre, C. R. Acad. Sci. Paris vol. 177 (1923) pp. 102-104.

${ }^{12}$ I. J. Schoenberg, Extensions of theorems of Descartes and Laguerre to the complex domain, Duke Math. J. vol. 2 (1936) pp. 84-94.

${ }^{13}$ S. Lipka, Über die Descartessche Zeichenregel, Acta Univ. Szeged. vol. 7 (19341935) pp. 177-185. 
the interval $\eta_{n} \leqq x<+\infty$.

The proof of the rule (6) is extremely simple and actually gives more since it turns out, as a matter of fact, that even the number of the positive sign changing places of $f(x)$ is not less than the number of sign changes of the sequence (6). It is based on the following theorem of Fejér. ${ }^{14}$

The number of the sign variations of a real function $g(x)$ in the interval $0<x<a(a \leqq+\infty)$ is not less than the number of sign changes of the sequence of moments:

$$
M_{0}=\int_{0}^{a} g(t) d t, \cdots, M_{n}=\int_{0}^{a} g(t) t^{n} d t .
$$

In order to prove our rule we form instead of the moments of $f(x)$ those of $f(x) e^{-x}$

$$
M_{k}=\int_{0}^{\infty} f(x) e^{-x} x^{k} d x, \quad k=0,1, \cdots, n .
$$

Owing to the expansion ${ }^{15}$

$$
x^{k}=k ! \sum_{\nu=0}^{k}(-1)^{\nu} C_{k, \nu} L_{\nu}(x)
$$

and the well known orthogonality property $^{16}$

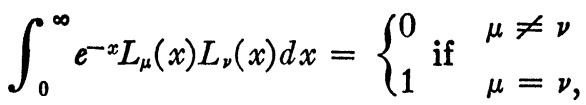

we obtain from (9)

$$
M_{k}=k ! \sum_{\nu=0}^{k}(-1)^{\nu} C_{k, \nu} b_{\nu}, \quad k=0,1, \cdots, n .
$$

${ }^{14}$ L. Fejér, Nombre des changement de signe d'une fonction dans un intervalle et ses moments, C. R. Acad. Sci. Paris vol. 158 (1914) pp. 1328-1331. If some of the moments are 0 , then we can of course simply drop them; we can get an improved lower estimation in the following way. Let, for example, $M_{\mu} \neq 0$. Let $c_{\mu}=M_{\mu}$; further for $\nu<\mu$, $c_{\nu}=M_{\nu}$ if $M_{\nu} \neq 0$ and $c_{\nu}=-\operatorname{sg} c_{\nu+1}$ if $M_{\nu}=0$; finally for $\nu>\mu, c_{\nu}=M_{\nu}$ if $M_{\nu} \neq 0$ and $c_{\nu}=-\operatorname{sg} c_{\nu-1}$ if $M_{\nu}=0$. Then the number of sign changes of $g(x)$ in $0<x<a$ is not less than even the number of sign changes in the sequence $c_{0}, c_{1}, \cdots, c_{n}$. See Pólya-Szegö, loc. cit. footnote 8 , vol. 2 , p. 50 .

${ }^{15}$ I don't know to whom this identity is due. I have seen it stated and proved in the paper of E. Feldheim, Contributions a la theorie des polynômes de Jacobi (Hungarian with French summary), Matematikai és Physikai Lapok vol. 48 (1941) pp. 453-504.

${ }^{16}$ See, for example, G. Szegö, Orthogonal polynomials, Amer. Math. Soc. Colloquium Publications, vol. 23, 1939, p. 96. 
This together with the quoted theorem of Fejer shows that the number of positive sign changing places of $f(x) e^{-x}$ (or of $f(x)$ ) is not less than the number of sign changes in the sequence (10) or, what amounts to the same, in the sequence (6).

Of course a similar reasoning yields similar rules for other polynomial expansions but the rule given for Laguerre expansion seems to be particularly simple and to a certain extent dual to the rule of Laguerre (3). The question of constructing such simple rules leads to interesting questions in the theory of orthogonal polynomials; to these I shall return elsewhere.

InSTITUTE For AdVANCEd STUdy 